\title{
ÍNDICE CINTURA-TALLA E ÍNDICE DE MASA CORPORAL COMO PREDICTORES DE RIESGO CARDIOMETABÓLICO EN NIÑOS Y ADOLESCENTES
}

\section{Waist size index and body mass index as predictors of cardiometabolic risk in children and adolescents}

\section{Ángela M. Matos Imberta, Jairo Blanco Jiménez ${ }^{\mathrm{b}}$, Xiomara Rodríguezc, Carina De La Motad, Alina Fernández Genao e, Noemí Acevedo Rodríguezf, Haydee Rochits Cuetog, oan M. Reynoso Rosarioh, Michelle M. Jiménez Azconai y Isi Y. Ortiz Hernandezi}

Recibido: 31 agosto, $2020 \bullet$ Aprobado: 30 octubre, 2020

Cómo citar: Matos Imbert Ángela M, Blanco Jiménez J, RodríguezX, De La Mota C, Fernández Genao A, Acevedo Rodríguez N, Rochits Cueto H, Reynoso Rosario JM, Jiménez Azcona MM, Ortiz Hernandez IY. Índice cintura-talla e índice de masa corporal como predictores de riesgo cardiometabólico en niños y adolescentes. cysa [Internet]. 11 de junio de 2021 [citado 16 de junio de 2021];5(2):77-5. Disponible en: https://revistas.intec.edu.do/index.php/cisa/article/view/2224

\section{Resumen}

Objetivo: comparar la utilidad del índice cintura-talla y el índice de masa corporal para detectar factores de riesgo cardiometabólicos en niños de 5-18 años, atendidos en la consulta externa del Hospital Infantil Regional Universitario Doctor Arturo Grullón en el período octubre-diciembre del año 2016.

Método: se realizó un estudio observacional, comparativo, de corte transversal y fuente primaria, con una muestra de 118 pacientes. Se midieron las variables sociodemográ-

\footnotetext{
a Médico Pediatra Endocrinóloga, Jefa Departamento de Endocrinología Pediátrica, Docente Cátedra Pediatría, Pontificia Universidad Católica Madre y Maestra (PUCMM). ORCID: 0000-0003-4009-6118, Correo-e: am.matos@ce.pucmm.edu.do

b Médico Pediatra, Hospital Infantil Regional Universitario Dr. Arturo Grullón. ORCID: 0000-0001-9027-5937

Correo-e: 20131592@ce.pucmm.edu.do

c Médico Pediatra, Hospital Infantil Regional Universitario Dr. Arturo Grullón. ORCID: 0000-0003-3001-2259,

Correo-e:20131595@ce.pucmm.edu.do

d Médico Pediatra, Hospital Infantil Regional Universitario Dr. Arturo Grullón. ORCID: 0000-0003-4966-6738

Correo-e: 20131584@ce.pucmm.edu.do

'Estudiante Medicina, PUCMM. ORCID: 0000-0002-5102-8252, Correo-e: 20150088@ce.pucmm.edu.do
}

\begin{abstract}
Objective: To compare the utility of the WSI and BMI in detecting cardiometabolic risks on children between 5-18 years, during external consult in Hospital Infantil Regional Universitario Dr. Arturo Grullón in the period of October-December 2016.

Methods: A cross-sectional, primary source, observational design study was conducted with a sample of 118 patients. The variables measured for this study included sociodemographic (age and sex), anthropometric (weight, height,

\footnotetext{
${ }_{\mathrm{f}}$ Médico General. Docente Cátedra Investigación, PUCMM. ORCID: 0000-0001-5853-8389, Correo-e: n.acevedo@ce.pucmm.edu.do g Médico General. Docente Cátedra Investigación, PUCMM. ORCID: 0000-0002-2920-7377, Correo-e: h.rochits@ce.pucmm.edu.do,

${ }^{\text {h }}$ Médico General, PUCMM. ORCID: 000-0003-2104-8508 Correo-e: 20141124@ce.pucmm.edu.do

${ }^{i}$ Médico Internista, PhD Salud Comunitaria. Docente e Investigadora, PUCMM. ORCID: 0000-0002-1464-5294

Correo-e: mm.jimenez@ce.pucmm.edu.do

¡ Médico Pediatra del Hospital Infantil Regional Universitario Dr. Arturo Grullón. Docente e Investigadora. PUCMM. Miembro del Comité de Investigación de Medicina PUCMM-STI.

ORCID: 0000-0002-6906-4016, Correo-e: iy.ortiz@ce.pucmm.edu.do
} 
ficas (edad y sexo), antropométricas (peso, talla, IMC, ICT, PC, TA), y laboratorios (glicemia, ALT, colesterol, triglicéridos, HDL, LDL). Para el análisis cuantitativo se calculó el promedio y la desviación estándar, para el análisis cualitativo se utilizó la prueba estadística chi-cuadrado.

Resultados: tanto el ICT como el IMC detectan de manera similar las alteraciones de la presión arterial sistólica $(\mathrm{ICT}=15.9 \%, \mathrm{IMC}=15 \%)$, diastólica $(\mathrm{ICT}=20.4 \%$, $\mathrm{IMC}=21.8 \%)$, obesidad $(\mathrm{ICT}=69.5 \%$, IMC=73.7 \%), HDL (ICT $=6.8 \%, \mathrm{IMC}=5.6 \%)$. En relación a la evaluación de la obesidad ambos índices arrojaron resultados afines, 69.5 \% para ICT y 73.7 \% el IMC. En la valoración de los niveles altos de ALT se obtuvieron resultados similares, presentando el ICT un $8.1 \%$ y el IMC un $8.9 \%$.

Conclusión: se demostró que tanto el ICT como el IMC son buenos predictores de factores de riesgos cardiometabólicos.

Palabras claves: relación cintura-estatura; índice de masa corporal; circunferencia de la cintura; medición de riesgo; síndrome metabólico; salud del niño.

\section{Introducción}

La infancia y adolescencia son etapas caracterizadas por un crecimiento y desarrollo físico. Los hábitos adquiridos en este período pueden condicionar la calidad de vida a largo plazo, aumentando la posibilidad del desarrollo de sobrepeso y obesidad infantil, influenciado por factores como el estilo de vida sedentario y el desequilibrio en la ingesta alimentaria. Se ha visto que los individuos que padecen de sobrepeso durante la infancia tienen una mayor tendencia a ser obesos en la vida adulta ${ }^{1}$, trayendo como consecuencia un aumento en el riesgo de padecer enfermedades cardiovasculares, diabetes mellitus, enfermedades pulmonares obstructivas, esteatosis hepática, problemas en las articulaciones, entre otros ${ }^{2}$. De manera similar, se han identificado consecuencias psicológicas de la obesidad como son la ansiedad, depresión, baja autoestima y percepción de la calidad de vida disminuida ${ }^{3}$. La obesidad infantil representa un problema de salud pública a nivel mundial, se estima que 41 millones de niños
BMI, ICT, PC), and laboratories (glycemia, ALT, cholesterol, triglycerides, HDL, LDL). The quantitative analysis was carried out by calculating the average and the standard deviation. As for the qualitative analysis, the statistical test $\chi^{2}$ was used.

Results: Both the WSI and the BMI proved to be effective in detecting changes in Systolic (WSI $=15.9 \%, \mathrm{BMI}=$ 18.4\%) and Diastolic Blood Pressure (WSI $=20.4 \%$, BMI $=18.6 \%$ ), obesity $(\mathrm{WSI}=69.5 \%, \mathrm{BMI}=73.7 \%), \mathrm{HDL}$ (WSI $=29.3 \%, \mathrm{BMI}=29.9 \%)$. Regarding the detection of obesity, both rates showed similar results, $69.5 \%$ for ICT, and $73.7 \%$ for BMI. Comparable results were obtained in the assessment of high levels of ALT, with the ICT at $8.1 \%$ and the BMI at $8.9 \%$.

Conclusions: WSI and BMI are both useful to detect cardiometabolic risk factors.

Keywords: Waist-Height Ratio; Body Mass Index; Waist Circumference; Risk Assessment; Metabolic Syndrome; Child Health.

menores de 5 años fueron reportados con sobrepeso u obesidad en $2016^{4}$. En ese mismo año, aproximadamente 340 millones de niños y adolescentes, entre las edades de 5 a 19 años, cursaron con sobrepeso u obesidad ${ }^{4}$.

La República Dominicana es uno de los países con índice de obesidad más alto en la región del Caribe, donde un $30 \%$ de la población es considerada obesa ${ }^{5}$. En el año 2013, la prevalencia de obesidad fue de $7.30 \%$ para niñas y $7.90 \%$ para niños menores de 5 años ${ }^{6}$. A nivel infantil existe un $6.5 \%$ de sobrepeso solamente en menores de cinco años ${ }^{7}$, lo que representa un problema social que debe atenderse. En la práctica clínica pediátrica se utilizan índices clínicos y metabólicos para medir el aumento de la masa celular, tisular y corporal desde el periodo de la lactancia hasta la pubertad. Dichos índices toman en cuenta parámetros como el peso (kg) y la talla (mt) para la edad, los cuales son accesibles y de bajo costo, permitiendo detectar a tiempo irregularidades ${ }^{8}$. 
La relación cintura-talla representa una herramienta sencilla y no invasiva que podría ser utilizada para el diagnóstico de obesidad, y como método eficaz en la detección de factores de riesgo cardiometabó$\operatorname{licos}^{9,10}$. Debido a que las herramientas que existen actualmente son complejas y requieren tiempo para el pediatra, ya que la mayoría deben utilizar tablas con valores de referencia para cada edad, se necesita implementar una herramienta útil, eficaz y sencilla, como lo es la razón cintura/talla, para la determinación con un único punto de corte para cada sexo y edad, y que sea capaz de diagnosticar obesidad central y estratificar el riesgo cardiometabólico ${ }^{10}$. Por lo que el presente estudio tuvo por propósito comparar la utilidad del índice cintura talla y el índice de masa corporal para detectar factores de riesgo cardiometabólicos.

\section{Materiales y métodos}

Se llevó a cabo un estudio de diseño observacional de tipo comparativo, de corte transversal y de fuente primaria. La población de estudio estuvo comprendida por los pacientes de 5 a 18 ańos que acudieron a la consulta externa de nutrición y endocrinología del Hospital Infantil Dr. Arturo Grullón entre los meses de octubre-diciembre del año 2016. Se utilizó un muestreo tipo probabilístico, aleatorio simple con sustitución, a través de la lista de los pacientes citados a consulta. Para el cálculo muestral se utilizó la página http://www. raosoft.com/samplesize.html, bajo los parámetros de $95 \%$ para el nivel de confianza, margen de error $5 \%$, resultando una muestra de 118 nińos. Para la selección de los participantes en esta investigación, se tomaron en cuenta como criterios de inclusión: niños con edad entre 5-18 años, y la firma del asentimiento informado y/o consentimiento informado según aplicare el caso de acuerdo con la edad. Se excluyeron del estudio aquellos pacientes que estaban en tratamiento con esteroides, con deformidades en la columna vertebral y/o con patología tiroidea o renal.
Las variables de análisis fueron clasificadas en: sociodemográficas (edad y sexo), antropométricas (peso, talla, IMC, ICT, PC, TA), bioquímicas (glicemia, ALT, colesterol, triglicéridos, HDL, LDL).

La validación del instrumento y el procedimiento de recolección de datos se realizó a través de una prueba piloto externa, en las consultas de Endocrinología y Nutrición del Hospital Infantil Regional Universitario Dr. Arturo Grullón (HIRUDAG), con un total de 14 pacientes, lo cual correspondió al $10 \%$ de la muestra calculada. El procedimiento de recolección de datos para el análisis de datos final se llevó a cabo en un periodo de 3 meses sobre una muestra de 114 pacientes seleccionados, en el centro hospitalario mencionado anteriormente, dentro del horario de 8:00 am - 12:00 pm.

Para las medidas antropométricas analizadas, se utilizó la balanza Kenwell (PM670) para medir el peso, la misma se calibró antes de pesar a cada participante. Se registró la talla en metros con una barra rígida para evitar errores en la medición. Asimismo, se tomó la presión arterial, y se obtuvo el índice de masa corporal por medio de la fórmula de cociente entre el peso en kilogramos y la talla en metros cuadrados. El valor resultante se extrapoló a las gráficas de crecimiento en percentiles de índice de masa corporal para edad y sexo de la OMS 2007. Se obtuvo índice cintura-talla, mediante la toma del perímetro cintura tomando como lugar la medición el punto entre la cresta iliaca y la última costilla, mediante una cinta métrica flexible, anotando en $\mathrm{cm}$ y $\mathrm{mm}$, entre la obtención de la talla como se detalló anteriormente, el cálculo se efectuó al dividir ambas medidas. Por último, se consultó el historial clínico del paciente de donde se tomaron los resultados de las pruebas analíticas correspondiente y en dado caso de no estar todas las pruebas analíticas en el expediente se hizo la indicación para su realización en el laboratorio del hospital, como son la glicemia, colesterol, triglicéridos, transaminasas hepáticas, la cuales son parte de las pruebas protocolizadas en las consultas de endocrinología y nutrición. 
La base de datos fue creada y procesada en Microsoft Excel 2013, tras lo cual se exportó para su tratamiento estadístico al programa IBM SPSS Statistics 23. La prueba estadística inferencial utilizada para las asociaciones estadísticas fue Chi Cuadrado.

\section{Consideraciones bioéticas}

Con la finalidad de proteger los principios bioéticos, los participantes y sus tutores fueron informados del objetivo y procedimiento del estudio. En ese mismo orden, fueron respondidas todas las preguntas de lugar. Una vez agotado este paso, se solicitó el consentimiento informado a los padres y tutores, así como el asentimiento informado a los niños y niñas mayores de 7 años. Cabe destacar que la propuesta de investigación fue evaluada y aprobada por el Comité de Bioética de la Facultad de
Ciencias de la Salud de la Pontificia Universidad Católica Madre y Maestra (COBE-FACS) antes de su ejecución.

\section{Análisis de los resultados}

Al comparar el IMC y el ICT en relación con la edad de los participantes: de los 118 nińos estudiados se observó un ICT > 0.51 en un $15.3 \%$ entre las edades de 5 a 8 años, un $12.7 \%$ entre 9 a 13 años. Se vislumbró que $36.4 \%$ de los niños entre 9 a 13 años presentó valores de IMC mayores al percentil 85 , con sobrepeso y obesidad. Los valores de ICT > 0.51 se observaron predominantemente en el sexo masculino para un $16.9 \%$, en comparación con el $12 \%$ en el sexo femenino. Con relación al IMC por encima del percentil 85, se registró un $39 \%$ de los participantes de sexo masculino. (Véase tabla 1)

Tabla 1. Características sociodemográficas en relación al índice cintura talla e índice masa corporal como predictores de riesgo cardiometabólico en nińos y adolescentes. $\mathrm{N}=114$

\begin{tabular}{|c|c|c|c|c|c|c|c|c|c|}
\hline \multicolumn{2}{|c|}{ Variables } & \multicolumn{4}{|c|}{ ICT } & \multicolumn{4}{|c|}{ IMC } \\
\hline & & \multicolumn{2}{|c|}{$<0,50$} & \multicolumn{2}{|c|}{$>0,51$} & \multicolumn{2}{|c|}{$<84$ th } & \multicolumn{2}{|c|}{$>85$ th } \\
\hline & & $\mathrm{n}$ & $\% *$ & $\mathrm{n}$ & $\% *$ & $\mathrm{n}$ & $\% *$ & $\mathrm{n}$ & $\% *$ \\
\hline \multirow[t]{3}{*}{ Edad } & 5-8 años & 18 & 15,3 & 23 & 19,5 & 15 & 12,7 & 26 & 22 \\
\hline & $9-13$ años & 15 & 12,7 & 43 & 36,4 & 15 & 12,7 & 43 & 36,4 \\
\hline & $14-18$ años & 3 & 2,5 & 16 & 13,6 & 1 & 0,9 & 18 & 15,3 \\
\hline \multirow[t]{2}{*}{ Sexo } & Masculino & 20 & 16,9 & 46 & 39 & 15 & 12,7 & 51 & 43,2 \\
\hline & Femenino & 16 & 13,6 & 36 & 30,5 & 16 & 13,6 & 36 & 30,5 \\
\hline
\end{tabular}

Fuente: instrumento de recolección de datos. * Porcentajes de casillas calculados por el total de la muestra.

80 | Ciencia y Salud 2021; 5(2, mayo-agosto): 77-85 • Artículo original 
El análisis del IMC y el ICT en relación con los niveles de presión arterial sistólica (PAS) de los participantes: se observó en el grupo con un ICT > 0.51 que el $81.7 \%$ presentó niveles de tensión arterial sistólica normales para la edad, un $18.3 \%$ con niveles elevados. Se demostró que $76.8 \%$ de los niños con IMC mayores al percentil 85 presentaron niveles de PAS normales para la edad, mientras que el $23.2 \%$ tuvo niveles elevados. En cuanto a los niveles de presión arterial diastólica (PAD) se observó la tendencia de niveles normales en el $81.6 \%$ de los participantes con IMC mayores al percentil 85, en comparación con el $78.2 \%$ del grupo con ICT $>0.51$. (Véase tabla 2)

La evaluación de los niveles de glicemia en relación con el ICT $>0.51 \mathrm{~cm}$, denota que el $91.5 \%$ de los participantes presentó niveles de glicemia normal, en comparación con $8.5 \%$ con niveles elevados. Al compararlos con los individuos con IMC mayores al percentil 85, el $93.1 \%$ tuvo valores normales de glicemia y solo se observó un $6.9 \%$ con valores por encima de la normalidad. (Véase tabla 2)

Al comparar el IMC y el ICT en relación con los niveles de ALT de los participantes: se observó en el grupo con un ICT > 0.51 que el $78 \%$ presentó niveles en el rango normal, un $22 \%$ con niveles elevados. Se objetivó que $93.1 \%$ de los niños con IMC $>85$ th, presentó niveles de ALT normales para la edad, mientras que el $6.9 \%$ objetivó niveles elevados. En cuanto a los niveles de colesterol total se observó la tendencia de niveles normales en el 61 $\%$ de los participantes con IMC $>85$ th, en comparación con el $59.8 \%$ del grupo con ICT > 0.51 .

$\mathrm{Al}$ analizar el IMC y el ICT en relación con los niveles de colesterol LDL de los participantes: se observó en el grupo con un ICT $>0.51$ que el $76.8 \%$ presentó niveles óptimos, un $12.2 \%$ en el rango límite y un $11 \%$ con valores elevados. Se objetivó que $78.2 \%$ de los niños con IMC > 85 th, presentó niveles en el rango de la normalidad, mientras que el $21.8 \%$ se encontró por encima del rango normal. En cuanto a los niveles colesterol HDL se observó la tendencia de niveles normales en el $70.1 \%$ de los participantes con IMC $>85$ th, en comparación con el $70.7 \%$ del grupo con ICT $>0.51$. (Véase tabla 2)

$\mathrm{Al}$ evaluar el IMC y el ICT en relación con los niveles de triglicéridos de los nińos objeto de estudio, se observó en el grupo con un ICT $>0.51$ que el $47.6 \%$ presentó niveles de triglicéridos adecuados, en comparación con un $52.4 \%$ con niveles elevados. Se objetivó que $49.4 \%$ de los niños con IMC > 85 th, presentó niveles normales, mientras que el $50.6 \%$ observó niveles elevados. (Véase tabla 2) 
Tabla 2. Índice cintura talla, índice masa corporal y perímetro cintura como predictores de riesgo cardiometabólico en niños y adolescentes

\begin{tabular}{|c|c|c|c|c|c|c|c|c|c|c|c|c|}
\hline \multirow[t]{3}{*}{ Variables } & \multicolumn{4}{|c|}{ Índice cintura talla } & \multicolumn{4}{|c|}{ IMC } & \multicolumn{4}{|c|}{ Perímetro cintura } \\
\hline & \multicolumn{2}{|c|}{$<0.5$} & \multicolumn{2}{|c|}{$>0.51$} & \multicolumn{2}{|c|}{$<84$ th } & \multicolumn{2}{|c|}{$>85$ th } & \multicolumn{2}{|c|}{$<74$ th } & \multicolumn{2}{|c|}{$>75$ th } \\
\hline & $\mathbf{n}$ & $\% *$ & $\mathbf{n}$ & $\% *$ & $\mathbf{n}$ & $\% *$ & $\mathbf{n}$ & $\% *$ & $\mathbf{n}$ & $\% *$ & $\mathbf{n}$ & $\% *$ \\
\hline $\begin{array}{c}\text { PAS }^{* *} \\
\text { Normal }\end{array}$ & 35 & 97,2 & 67 & 81,7 & 31 & 100 & 71 & 81,6 & 30 & 25,4 & 72 & 61 \\
\hline Normal-alta & 1 & 2,8 & 12 & 14,6 & 0 & 0 & 13 & 14,9 & 0 & 0 & 13 & 11 \\
\hline Estadio 1 & 0 & 0 & 1 & 2,4 & 0 & 0 & 1 & 1,2 & 0 & 0 & 1 & 0,9 \\
\hline Estadio 2 & 0 & 0 & 2 & 1,7 & 0 & 0 & 2 & 2,3 & 0 & 0 & 2 & 1,7 \\
\hline $\begin{array}{c}\text { PAD } \\
\text { Normal }\end{array}$ & 35 & 97,2 & 63 & 76,8 & 30 & 96,8 & 68 & 78,2 & 29 & 24,6 & 69 & 58,5 \\
\hline Normal-alta & 1 & 2,8 & 15 & 18,3 & 1 & 3,2 & 15 & 17,2 & 1 & 0,8 & 15 & 12,7 \\
\hline Estadio 1 & 0 & 0 & 3 & 3,7 & 0 & 0 & 3 & 3,5 & 0 & 0 & 3 & 2,6 \\
\hline Estadio 2 & 0 & 0 & 1 & 1,2 & 0 & 0 & 1 & 1,1 & 0 & 0 & 1 & 0,8 \\
\hline Glicemia Normal & 30 & 83,3 & 75 & 91,5 & 24 & 77,5 & 81 & 93,1 & 24 & 20,4 & 81 & 68,6 \\
\hline Alta & 6 & 16,7 & 7 & 8,5 & 7 & 22,5 & 6 & 6,9 & 6 & 5,1 & 7 & 5,9 \\
\hline $\begin{array}{c}\text { ALT } \\
\text { Normal }\end{array}$ & 31 & 86,1 & 64 & 78 & 27 & 87,1 & 68 & 78,2 & 27 & 22,9 & 68 & 57,6 \\
\hline Alta & 5 & 13,9 & 18 & 22 & 4 & 12,9 & 19 & 21,8 & 3 & 2,6 & 20 & 16,9 \\
\hline $\begin{array}{c}\text { LDL } \\
\text { Óptimo }\end{array}$ & 30 & 83,3 & 63 & 76,8 & 25 & 80,6 & 68 & 78,2 & 27 & 22,9 & 66 & 55,9 \\
\hline Límite & 4 & 11,1 & 10 & 12,2 & 4 & 12,9 & 10 & 11,5 & 2 & 1,7 & 12 & 10,2 \\
\hline Alto & 2 & 5,6 & 9 & 11 & 2 & 6,5 & 9 & 10,3 & 1 & 0,8 & 10 & 8,5 \\
\hline Colesterol Deseable & 24 & 66,7 & 49 & 59,8 & 20 & 64,5 & 53 & 61 & 22 & 18,6 & 51 & 43 \\
\hline Límite & 8 & 22,2 & 21 & 25,6 & 6 & 19,4 & 23 & 26,4 & 7 & 5,9 & 22 & 19 \\
\hline Alto & 4 & 11,1 & 12 & 14,6 & 5 & 16,1 & 11 & 12,6 & 1 & 0,8 & 15 & 12,7 \\
\hline $\begin{array}{c}\text { HDL } \\
\text { Baja }\end{array}$ & 13 & 36,1 & 24 & 29,3 & 11 & 35,5 & 26 & 29,9 & 12 & 10,2 & 25 & 21,2 \\
\hline Deseable & 23 & 63,9 & 58 & 70,7 & 20 & 64,5 & 61 & 70,1 & 18 & 15,3 & 63 & 53,3 \\
\hline Triglicéridos Adecuado & 23 & 63,9 & 39 & 47,6 & 19 & 61,3 & 43 & 49,4 & 23 & 19,5 & 39 & 33,1 \\
\hline Alto & 13 & 36,1 & 43 & 52,4 & 12 & 38,7 & 44 & 50,6 & 7 & 5,9 & 49 & 41,5 \\
\hline
\end{tabular}

Fuente: instrumento de recolección de datos. PAS: Presión Arterial Sistólica, PAD: Presión Arterial Diastólica

*Porcentajes totales de casillas por columnas.

**Normal < 90th; Normal-alto 90th-95th, Hipertensión Estadio $1>95-99$ más 5 mmHg, Hipertensión Estadio $2>99$ más $5 \mathrm{mmHg}$.

82 | Ciencia y Salud 2021; 5(2, mayo-agosto): 77-85 • Artículo original 


\section{Discusión}

Al comparar el ICT e IMC y su utilidad para detectar factores de riesgo cardiometabólico, se evidenció que tanto el ICT como IMC detectaron de forma similar los niveles alterados de PAS (ICT $=15.9 \% \mathrm{e}$ $\mathrm{IMC}=18.4 \%)$, PAD (ICT=20.4\% e IMC=18.6\%), glicemia (ICT=8.5\% e IMC=6.9\%) y obesidad (ICT=69.5 \% e IMC=73.7 \%). Sin embargo, el ICT se encontró asociado con mayor frecuencia a niveles elevados de LDL (ICT=6.5 \% e IMC=2.4\%), colesterol (ICT=6.9 \% e IMC=3.5\%) y triglicéridos ( $\mathrm{ICT}=16.3 \%$ e $\mathrm{IMC}=11.9 \%$ ). Estos resultados indican que ambos perímetros detectan de igual manera factores de riesgo cardiometabólico en niños. De forma similar, Arnaiz et al. ${ }^{11}$ comparó el índice cintura-talla (ICT) e índice masa corporal (IMC) como predictores del síndrome metabólico, exponiendo que el ICT mostró similares resultados en cuanto a la sensibilidad y especificidad al comparar con el IMC. Por su parte, Mansur et al. ${ }^{12}$ evidenció la correlación positiva del ICT y el IMC reportando un valor de $\mathrm{P}<0.00$, concluyendo que ambos índices son de gran utilidad para la detección de factores de riesgo metabólico.

Al determinar la relación entre los factores de riesgo con ICT y IMC según la edad y el sexo, el sexo que se relacionó con mayor alteración del ICT fue el masculino con un $39 \%$ y en el IMC con un $43.2 \%$. Además, la edad que presentó más relación predictiva con el ICT e IMC fue 9-13 años, con un $36.4 \%$ y un $36.4 \%$, respectivamente. Al comparar con el estudio Arnaiz et al. ${ }^{13}$ que determinó cómo se relaciona al ICT, la edad predominante fue de 9,86 $\pm 2,33$ ańos, sin diferencias por sexo $(\mathrm{p}=0.531)$, concluyendo que la utilización ICT no varía con la edad, sexo o maduración puberal. Serrano et al. ${ }^{14}$ analizaron la asociación entre el índice cintura-talla y la grasa corporal para el diagnóstico de obesidad infantil, encontrando como resultados que el sexo femenino fue el relacionado con sobrepeso y obesidad. Los estudios previamente mencionados muestran que el sexo femenino tuvo una mayor relación con la obesidad, a diferencia de lo observado en la presente investigación. Dicha oposición en los resultados puede estar relacionada a que la población femenina representaba más de un $50 \%$ de la muestra en los estudios anteriores, mientras que en el presente representó un $44.1 \%$. Otro punto que destacar en la diferencia de algunas conclusiones, es el tamaño de la muestra $(n>2000)$ de los estudios citados, en comparación con esta investigación donde la muestra fue de 118 casos.

Al comparar la relación entre el ICT y el IMC con los niveles de presión arterial, los resultados obtenidos fueron que el ICT > $0.51 \mathrm{~cm}$ mostró un $15.9 \%$ de presentar alteración de los niveles de PAS y el IMC $>85$ th mostró un $18.4 \%$, al respecto de la PAD, el ICT encontró un $20.4 \%$ y el IMC fue $18.6 \%$ para detectar PAD alterada, lo que nos muestra que ambos parámetros son de utilidad para el riesgo de la presión arterial. En otra investigación realizada por Chen et al. ${ }^{15}$, en el 2012, acerca de la asociación del índice cintura talla y la hipertensión arterial en niños, usando el punto de corte de este índice de 0.5 mostraron una prevalencia de hipertensión arterial de un $21.2 \%$, concluyendo así, que el índice cintura-talla es un factor importante asociado a hipertensión arterial.

\section{Conclusión}

Tanto el ICT como el IMC tienen un valor predictivo para detectar de manera similar las alteraciones de la PAS (ICT $=15.9 \%$, IMC=18.4\%), PAD (ICT $=20.4 \%$, IMC=18.6\%), obesidad (ICT=69.5\%, IMC=73.7 \%), HDL (ICT=29.3\%, IMC=29.9\%), glicemia (ICT=8.5 \%, IMC=6.9\%). El ICT tuvo más relación para la alteración del colesterol en un $6.9 \%$, los triglicéridos en un $16.3 \%$, LDL en un $6.5 \%$. El PC presentó mayor relación para detectar niveles alterados de LDL en un 16,2 \%, colesterol en $25 \%$, triglicéridos en $47.4 \%$. En la valoración de los niveles alto de ALT, ambos índices tuvieron casi los mismos resultados presentando el ICT un $22 \%$ y el IMC un $21 \%$. 


\section{Bibliografía}

1. Sobrepeso y obesidad infantiles [Internet]. Organización Mundial de la Salud. 2020 [citado 29 agosto 2020]. Disponible en: https://www. who.int/dietphysicalactivity/childhood/es/

2. Causes and Consequences of Childhood Obesity [Internet]. Centers for Disease Control and Prevention. 2020 [citado 29 agosto 2020]. Disponible en: https://www.cdc.gov/obesity/ childhood/causes.html

3. Rankin J, Matthews L, Cobley S, Han A, Sanders $\mathrm{R}$, Wiltshire $\mathrm{H}$ et al. Psychological consequences of childhood obesity: psychiatric comorbidity and prevention; 2016. Doi: 10.2147/AHMT. S101631

4. OMS. Obesidad y Sobrepeso. [Online]. [citado 10 marzo 2015]. Disponible en: http://www. who.int/mediacentre/factsheets/fs311/es/.

5. Amancio O, Ortigoza J, Durante I. Seminario EL Ejercicio Actual de la Medicina. [Online]; 2007 [citado 10 marzo 2015]. Disponible en: http:// www.facmed.unam.mx/sms/seam $2 \mathrm{k} 1 / 2007 /$ may_01_ponencia.html

6. República Dominicana - Obesidad infantil. https://www.indexmundi.com/es/datos/rep\%C3\%BAblica-dominicana/obesidad-infantil. Published 2016. Accessed May 16, 2020

7. Vásquez-Ruíz HA. ¿Es la obesidad un problema en República Dominicana? [Online]; 2014 [citado 10 marzo 2015]. Disponible en: https:// empirica.do/2740/es-la-obesidad-un-problema-en-republica-dominicana\#: : :text $=\mathrm{M} \%$ C3\%A1s\%20a\%C3\%BAn\%2C\%20el\%20 nivel\%20de, difiere\%20seg\%C3\%BAn\%20 los $\% 20$ estratos $\% 20$ poblacionales.
8. About Child and Teen BMI [Internet]. Centers for Disease Control and Prevention; 2020 [citado 1 septiembre 2020]. Disponible en: https://www. cdc.gov/healthyweight/assessing/bmi/childrens_ bmi/about_childrens_bmi.html

9. Gregorio Hernández J. Antropometrías de los Niños y Adolescentes. [Online]; 2012. Disponible en: http://medidasantropometricas.blogspot. com/2012_10_01_archive.html

10. Hernández J, Duchi P. Índice cintura/talla y su utilidad para detectar riesgo cardiovascular y metabólico. Rev Cubana Endocrinol. 2015;26(1). Disponible en: http://scielo.sld. $\mathrm{cu} /$ scielo.php?script $=$ sci_arttext\&pid=S1561$29532015000100006 \& \operatorname{lng}=$ es $\& \mathrm{nrm}=\mathrm{iso} \&$ tlng=es

11. Arnaiz P, Acevedo M, Díaz C, Bancalari R, Barja S, Aglony $\mathrm{M}$ et al. Razón cintura estatura como predictor de riesgo cardiometabólico en niños [Internet]. Rev Chil Cardiol. 2010;29:81-8. Disponible en: http://www. scielo.cl/scielo.php?script=sci_arttext $\&$ pid=S0 718-85602010000300001DOI:http://dx.doi. org/10.4067/S071885602010000300001 [citado febrero 2016].

12. Mansur V, Leone $C$, Damiani D. Is waist-toheight ratio a useful indicator of cardio-metabolic risk in 6-10-year-old children? BMC Pediatrics. 2013;13(91). Disponible en: https:// bmcpediatr.biomedcentral.com/articles/ 10.1186/1471-2431-13-91 DOI: https://doi. org/10.1186/1471-2431-13-91

13. Arnaiz P, Grob F, Cavada G, Domínguez A, Bancalari R, Cerda V. et al. La razón cintura estatura en escolares no varía con el género, la edad ni la maduración puberal. Rev. Med Chile. 2014;142:574-8.Disponible en: http://dx.doi. org/10.4067/S0034-98872014000500004. 
14. Serrano M, Cabañas M, Moreno M, González M, López N, Martínez J et al. Estimación de la adiposidad a partir del índice cintura talla: ecuaciones de predicción aplicables en población infantil española [Internet]. Nutrición Clínica. 2011;31(3):45-51 Disponible en: http://nutricion.org/publicaciones/revista_2011_03/ NUTRICION-31-3.pdf [citado 2 febr. 2016]
15. Chen T, Choy C, Chan W, Chen C, Liao C. Waist to height ratio and elevated blood pressure among children in Taiwan. Indian Pediatr. 2012 Jun;49(6):463-6. Disponible en: https:// doi.org/10.1007/s13312-012-0075-8 